\title{
Ecoepidemiological Model and Analysis of Prey-Predator System
}

\author{
Abayneh Fentie Bezabih $(D$, Geremew Kenassa Edessa, and Koya Purnachandra Rao
}

Department of Mathematics, Wollega University, Nekemte, Ethiopia

Correspondence should be addressed to Abayneh Fentie Bezabih; abaynehf@hu.edu.et

Received 16 November 2020; Revised 20 December 2020; Accepted 15 January 2021; Published 22 February 2021

Academic Editor: Alain Miranville

Copyright (C) 2021 Abayneh Fentie Bezabih et al. This is an open access article distributed under the Creative Commons Attribution License, which permits unrestricted use, distribution, and reproduction in any medium, provided the original work is properly cited.

\begin{abstract}
In this paper, the prey-predator model of five compartments is constructed with treatment given to infected prey and infected predator. We took predation incidence rates as functional response type II, and disease transmission incidence rates follow simple kinetic mass action function. The positivity, boundedness, and existence of the solution of the model are established and checked. Equilibrium points of the models are identified, and local stability analyses of trivial equilibrium, axial equilibrium, and disease-free equilibrium points are performed with the method of variation matrix and the Routh-Hurwitz criterion. It is found that the trivial equilibrium point $E_{o}$ is always unstable, and axial equilibrium point $E_{A}$ is locally asymptotically stable if $\beta k-\left(t_{1}\right.$ $\left.+d_{2}\right)<0, q p_{1} k-d_{3}(s+k)<0$ and $q p_{3} k-\left(t_{2}+d_{4}\right)(s+k)<0$ conditions hold true. Global stability analysis of an endemic equilibrium point of the model has been proven by considering the appropriate Lyapunov function. The basic reproduction number of infected prey and infected predators are obtained as $R_{01}=\left(q p_{1}-d_{3}\right)^{2} k \beta d_{3} s^{2} /\left(q p_{1}-d_{3}\right)\left\{\left(q p_{1}-d_{3}\right)^{2} k s\left(t_{1}+d_{2}\right)+r s q p_{2}\right.$ $\left.\left(k q p_{1}-k d_{3}-d_{3} s\right)\right\}$ and $R_{02}=\left(q p_{1}-d_{3}\right)\left(q p_{3} d_{3}\right) k+\alpha r s q\left(k q p_{1}-k d_{3}-d_{3} s\right) /\left(q p_{1}-d_{3}\right)^{2}\left(t_{2}+d_{4}\right) k$, respectively. If the basic reproduction number is greater than one, then the disease will persist in the prey-predator system. If the basic reproduction number is one, then the disease is stable, and if the basic reproduction number is less than one, then the disease dies out from the prey-predator system. Finally, simulations are done with the help of DEDiscover software to clarify results.
\end{abstract}

\section{Introduction}

Mathematical modeling of prey-predator systems of interaction of species have a long history since the original remarkable work done by the Lotka-Volterra Model in the 1920s [14], and the SIR model compartment of systems of population is another vital area of research after the pioneering work of Kermack and Mckendrick [1-9]. Anderson and May were the first who combined these two modeling systems, while Chattopadhyay and Arino were the first who used the term "ecoepidemiology" for such models $[2,3,6]$. The dynamics of disease in prey-predator systems now become an interesting area of research due to the fact that prey-predator interaction is rich and complex in nature [4, 6, 10-13]. Several mathematical models have been proposed and studied on prey-predator systems [1-6, 8-12]. Many studies focused on the study of disease in prey only $[1-3,5,6,10,12,14-$ $20]$, other researchers were interested in the study of disease within the predator population only $[18,21]$, and there are also some studies on diseases in both prey and predators [4, $8,11]$. In this paper, we proposed and studied infectious disease in both prey and predator interaction of species with treatment given to infected prey and infected predator.

\section{Mathematical Model Formulation and Assumptions}

In this paper, the prey-predator population is divided into five compartments. Let us denote $X(t)$ as the susceptible prey, $W(t)$ as the infected prey, $Y(t)$ as the susceptible predator, $Z(t)$ as the infected predator, and $H(t)$ as both infected prey and infected predator population under treatment. In the absence of infectious disease, the susceptible prey population grows logistically with intrinsic growth rate $r$ and environmental carrying capacity $k$, and only susceptible prey can reproduce. In the presence of infectious disease, susceptible predators become infected predators when they come into contact with infected predators, susceptible prey become 


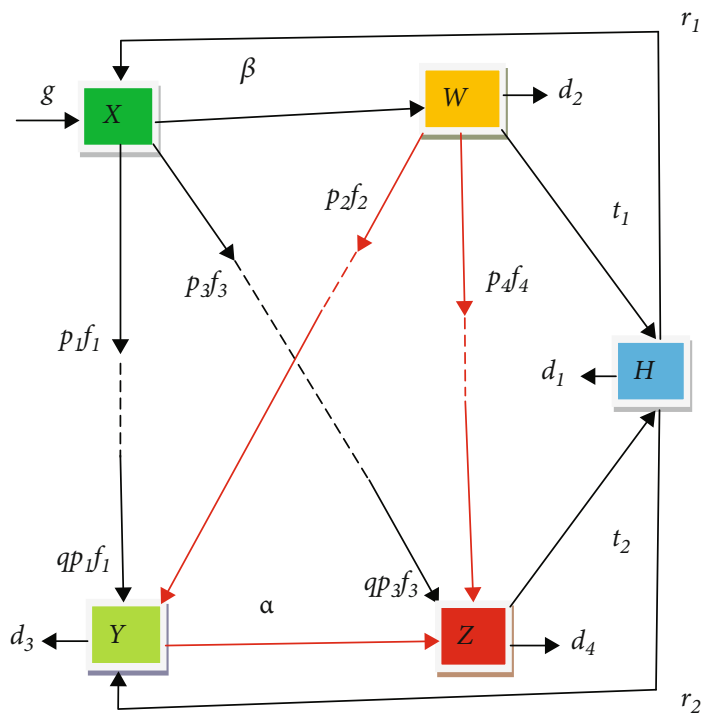

Figure 1: Model diagram.

infected prey when they come into contact with infected prey, and the contact process is assumed to follow bilinear functional with convolution rate $\alpha, \beta$, respectively. The predation functional response of predator towards the prey is assumed to follow a different Holling type II functional response form with $p_{1}, p_{2}$ respective predation coefficients of $X(t), W(t)$ due to susceptible predator, and $p_{3}, p_{4}$ respective predation coefficient of $X(t), Y(t)$ due to infected predator. Suppose consumed prey converted into predator with efficiency $q$ and also half-saturated constants. It is also assumed that infected prey $W(t)$ and infected predator $Z(t)$ can only recover through treatment and are treated at a treatment rate of $t_{1}, t_{2}$, respectively. The prey-predator population $H(t), W(t), Y(t)$, and $Z(t)$ suffers from infectious disease with death rate $d_{1}, d_{2}, d_{3}$, and $d_{4}$, respectively. Moreover, assume that all variables and parameters used in the model are nonnegative.

According to the above assumption, we have the following model flow diagram.

From the model flow diagram in Figure 1, we have the following set of differential equations:

$$
\frac{d X}{d t}=g(X, W)+r_{1} H-\beta X W-p_{1} f_{1}(X, Y)-p_{3} f_{3}(X, Z),
$$

$$
\frac{d W}{d t}=\beta X W-t_{1} W-d_{2} W-p_{2} f_{2}(W, Y)-p_{4} f_{4}(W, Z)
$$

$$
\begin{gathered}
\frac{d Y}{d t}=q p_{1} f_{1}(X, Y)+q p_{2} f_{2}(W, Y)+r_{2} H-\alpha Y Z-d_{3} Y, \\
\frac{d Z}{d t}=q p_{3} f_{3}(X, Z)+q p_{4} f_{4}(W, Z)+\alpha Y Z-t_{2} Z-d_{4} Z,
\end{gathered}
$$

$$
\frac{d H}{d t}=t_{1} W+t_{2} Z-d_{1} H-r_{1} H-r_{2} H,
$$

with initial conditions $X(0) \geq 0 ; W(0) \geq 0 ; Y(0) \geq 0 ; Z(0)$ $\geq 0 ; H(0) \geq 0$; and $p_{i}>0, i=1,2,3,4$, and $0<q \leq 1$.

Depending on the assumptions of per capita growth of function $g(X, W)$ for susceptible prey, and different type II functional responses $f_{i}, i=1,2,3,4$, we have a more feasible model (8) emanating from model (3) as

$$
\begin{aligned}
& \frac{d X}{d t}=r X\left(1-\frac{X+W}{k}\right)+r_{1} H-\beta X W-\frac{p_{1} X Y}{s+X}-\frac{p_{3} X Z}{s+X} \\
&=f(X, W, Y, Z, H), \\
& \frac{d W}{d t}=\beta X W-t_{1} W-d_{2} W-\frac{p_{2} W Y}{s+W}-\frac{p_{4} W Z}{s+W} \\
&=g(X, W, Y, Z, H), \\
& \frac{d Y}{d t}=q \frac{p_{1} X Y}{s+X}+q \frac{p_{2} W Y}{s+W}+r_{2} H-\alpha Y Z-d_{3} Y \\
&=h(X, W, Y, Z, H), \\
& \frac{d Z}{d t}=q \frac{p_{3} X Z}{s+X}+q \frac{p_{4} W Z}{s+W}+\alpha Y Z-t_{2} Z-d_{4} Z \\
&=i(X, W, Y, Z, H), \\
& \frac{d H}{d t}=t_{1} W+t_{2} Z-d_{1} H-r_{1} H-r_{2} H=j(X, W, Y, Z, H),
\end{aligned}
$$

with initial conditions $X(0) \geq 0 ; W(0) \geq 0 ; Y(0) \geq 0 ; Z(0)$ $\geq 0 ; H(0) \geq 0 ; p_{1}, p_{2}, p_{3}, p_{4}>0$, and $0<q \leq 1$, it is possible to arrange model (6)-(10) and express it in more compact forms with excellent patterns in

$$
\begin{aligned}
& \frac{d X}{d t}=X\left\{r\left(1-\frac{X+W}{k}\right)-\left(\beta W+\frac{p_{1} Y+p_{3} Z}{s+X}\right)\right\}+r_{1} H, \\
& \frac{d W}{d t}=W\left\{\beta X-\left(t_{1}+d_{2}+\frac{p_{2} Y+p_{4} Z}{s+W}\right)\right\}, \\
& \frac{d Y}{d t}=Y\left\{q\left(\frac{p_{1} X}{s+X}+\frac{p_{2} W}{s+W}\right)-\left(\alpha Z+d_{3}\right)\right\}+r_{2} H, \\
& \frac{d Z}{d t}=Z\left\{q\left(\frac{p_{3} X}{s+X}+\frac{p_{4} W}{s+W}\right)+\alpha Y-\left(t_{2}+d_{4}\right)\right\}, \\
& \frac{d H}{d t}=t_{1} W+t_{2} Z-\left(d_{1}+r_{1}+r_{2}\right) H,
\end{aligned}
$$

with initial conditions $X(0) \geq 0 ; W(0) \geq 0 ; Y(0) \geq 0 ; Z(0)$ $\geq 0 ; H(0) \geq 0 ; p_{1}, p_{2}, p_{3}, p_{4}>0$; and $0<q \leq 1$. 


\section{Mathematical Analysis of the Model}

In this section, positivity, boundedness, and existence of the solution of the model are checked. This mathematical analysis of the model could be considered as the primary results.

Theorem 1 (boundedness). All solutions of model (8) are bounded in feasible region $\mathbb{R}_{+}^{5}$.

Proof. Each solution $X(t), W(t), Y(t), Z(t)$, and $H(t)$ of the model is bounded if and only if total population $N$ is bounded. Let total population of prey-predator $N=X+W$ $+Y+Z+H$.

For $\Lambda>0$ to be constant,

$$
\frac{d N}{d t}+\Lambda N=\frac{d X}{d t}+\frac{d W}{d t}+\frac{d Y}{d t}+\frac{d z}{d t}+\frac{d H}{d t}+\Lambda N
$$

By substituting all model Equations (8) into (12) and removing all negative terms, we have the following results: $d N / d t+\Lambda N \leq r X+q\left(p_{1} X Y /(s+X)\right)+q\left(p_{2} W Y /(s+W)\right)+$ $q\left(p_{4} W Z /(s+W)\right)+q\left(p_{3} X Z /(s+X)\right)+\Lambda N=\mu$. Then, solving the differential inequality $d N / d t+\Lambda N \leq \mu$ yields $N(t) \leq$ $(\mu / \Lambda)\left(1-e^{-\Lambda t}\right)+N(0) e^{-\Lambda t}$ for $t \rightarrow \infty, N \rightarrow \mu / \Lambda$. We know that total prey-predator population is nonnegative, and hence, $0 \leq N(t) \leq \mu / \Lambda$. So we have invariant feasible region $\Omega=\left\{(X, W, Y, Z, H) \in \mathbb{R}_{+}^{5}: 0 \leq N(t) \leq \mu / \Lambda\right\}$. This proves the theorem and the model is mathematically well posed

Theorem 2 (positivity). All solutions of model (6)-(10) are positive.

Proof. To prove Theorem 2, we have to show that variables $X(t), W(t), Y(t), Z(t), H(t)$ of the models (8) are nonnegative $\forall t \geq 0$.

(i) Positivity of $X(t)$ : from the susceptible prey model in (8), $d X / d t=r X(1-(X+W) / k)+r_{1} H-\beta X W$ $-p_{1} X Y /(s+X)-p_{3} X Z /(s+X)$ without loss of generality. After removing all the positive terms from the right-hand side of the differential equation, we have the following differential inequality: $d X / d t \geq-\left(\left(r X^{2}+r X W\right) / k+\beta X W+\left(P_{1} X Y+P_{3} X Z\right)\right.$ $/(S+X))$ divide both sides by negative yields $-(d$ $X / d t) \leq\left(R X^{2}+R X W\right) / k+\beta X W+\left(p_{1} X Y+p_{3} X Z\right) /$ $(S+X)$. But it is also clear that the following inequality holds: $\left(r X^{2}+r X W\right) / k+\beta X W+\left(P_{1} X Y\right.$ $\left.+P_{3} X Z\right) /(S+X) \leq r X^{2}+r X W+\beta X W+p_{1} X Y+p_{3}$ $X Z=X\left(r X+r W+\beta W+p_{1} Y+p_{3} Z\right)$. Assume that $r W+\beta W+p_{1} Y+p_{3} Z=C$, then the differential inequality is reduced to $-(d X / d t) \leq X(r X+C)$. This inequality can be arranged for integration by partial fraction as $\int(1 / X(r X+C)) d X \geq \int-d t$, integrating the integral inequality $\int((1 / C) / X+(-\mathrm{r}$ /C) $/(r X+C)) d X \geq-\int d t$ will give us $(1 / C) \ln |X|$ $-(1 / C) \ln |r X+C| \geq-t+Q$, where $Q$ is integration constant. Using rules of logarithm, the inequality can be written as $\ln |X /(r X+C)| \geq-C t+C Q$.
Finally, solving for $X$ will give us $X(t) \geq A C e^{-C t} /($ $\left.1-r A e^{-C t}\right)$, for $A=e^{C Q}$. Therefore, $X(t)>0$ for 1 $-r A e^{-C t}>0$. That is, $X(t)$ is nonnegative for $t>$ $(1 / C) \ln (r A)$.

(ii) Positivity of $W(t)$ : from the infected prey model in (7), $d W / d t=\beta X W-t_{1} W-d_{2} W-p_{2} W Y /(s+W)$ $-p_{4} W Z /(s+W)$, without loss of original generality, after removing the positive term $(\beta \mathrm{XW})$. We obtain the following differential inequality: $d W / d t \geq-\left(t_{1} W\right.$ $\left.+d_{2} W+p_{2} W Y /(s+W)+p_{4} W Z /(s+W)\right)$ if and only if $-(d W / d t) \leq\left(t_{1} W+d_{2} W+p_{2} W Y /(s+W)\right.$ $\left.+p_{4} W Z /(s+W)\right)$. But it is clear that $t_{1} W+d_{2} W$ $+p_{2} W Y /(s+W)+p_{4} W Z /(s+W) \leq t_{1} W+d_{2} W+$ $p_{2} W Y+p_{4} W Z=\left(t_{1}+d_{2}+p_{2} Y+p_{4} Z\right) W \quad$ holds true. Now assume that $t_{1}+d_{2}+p_{2} Y+p_{4} Z=C$. Then, we have $-(d W / d t) \leq C W t$. Now applying integration yield $\ln |W| \geq-C t+Q$, where $Q$ is the integration constant, then solving for the variable $W(t)$ gives the equation $W(t) \geq e^{-C t+Q}$ which is the exponential function and positive at all times. Hence, $W(t)$ is positive.

(iii) Positivity of $Y(t)$ : from the susceptible predator model in (8), $d Y / d t=q\left(p_{1} X Y /(s+X)\right)+q\left(p_{2} W Y /(\right.$ $s+W))+r_{2} H-\alpha Y Z-d_{3} Y$, without loss of original generality, after removing all positive terms $\left(q\left(p_{1} X\right.\right.$ $\left.Y /(s+X))+q\left(p_{2} W Y /(s+W)\right)+r_{2} H\right)$, we obtain differential equation $d y / d t \geq-\left(\alpha z+d_{3}\right) y$. Then, applying integration by separation of variable method results, $\ln |y| \geq-\left(\alpha z+d_{3}\right) t+Q$, where $Q$ is integration constant, and solving for variable $Y(t)$ ,we obtain the solution $|y| \geq e^{-\left(\alpha z+d_{3}\right) t+Q}$. Therefore, $y(t) \geq e^{-\left(\alpha z+d_{3}\right) t+Q}$ is a positive exponential function; hence, $y(t)$ is positive.

(iv) Positivity of $Z(t)$ : from the infected predator model in $(9), d Z / d t=q\left(p_{2} W Z /(s+W)\right)+q\left(p_{3} X Z /(s+W)\right.$ )$+\alpha Y Z-t_{2} Z-d_{4} Z$ after removing all positive terms $\left(q\left(p_{2} W Z /(s+W)\right)+q\left(p_{3} X Z /(s+W)\right)\right)$, we obtain the differential inequality $d z / d t \geq-\left(t_{2}+d_{4}\right) z$. Applying integration by separation of variable method yields $\ln |z| \geq-\left(t_{2}+d_{4}\right) t+Q$ where $Q$ is integration constant by separation of variable method. Then, solving for $Z$ will result in $z(t) \geq$ $e^{-\left(t_{2}+d_{4}\right) t+Q}$ which is the exponential function that is positive at all time. Hence, $Z(t)$ is positive.

Alternative verification: in mode (6)-(10), $d Z / d t=q\left(p_{2}\right.$ $W Z /(s+W))+q\left(p_{3} X Z /(s+W)\right)+\alpha Y Z-t_{2} Z-d_{4} Z$ Can be written as $d Z / d t=\left(q\left(p_{2} W /(s+W)\right)+q\left(p_{3} X /(s+W)\right)+\alpha Y\right.$ $\left.-t_{2}-d_{4}\right) Z$ if only if $(1 / Z) d Z=\left(q\left(p_{2} W /(s+W)\right)+q\left(p_{3} X /(\right.\right.$ $\left.s+W))+\alpha Y-t_{2}-d_{4}\right) d t$. This equation can be arranged as $\int(1 / Z) d Z=\int\left(q\left(p_{2} W /(s+W)\right)+q\left(p_{3} X /(s+W)\right)+\alpha Y-t_{2}\right.$ $\left.-d_{4}\right) d t$, which after computing the integration yields, $\ln \mid Z$ $\mid=\left(q\left(p_{2} W /(s+W)\right)+q\left(p_{3} X /(s+W)\right)+\alpha Y-t_{2}-d_{4}\right) t+Q$, where $Q$ is the integration constant. Thus, $Z(t)=$ $e^{\left(q\left(p_{2} W /(s+W)\right)+q\left(p_{3} X /(s+W)\right)+\alpha Y-t_{2}-d_{4}\right) t+Q}$ is the exponential function which is positive, and hence, $Z(t)$ is positive. 
TABle 1: Partial derivatives.

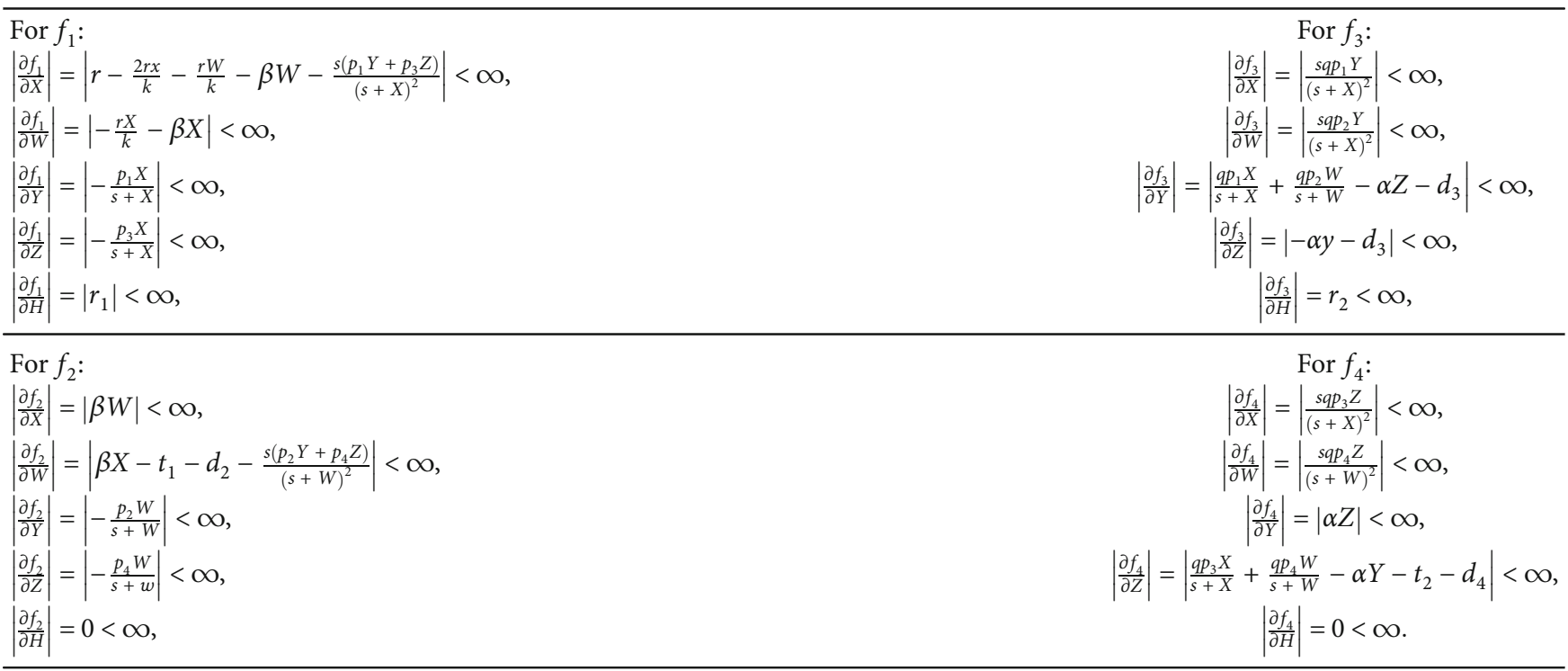

For $f_{5}$ :

$\left|\frac{\partial f_{5}}{\partial X}\right|=0<\infty$,

$\left|\frac{\partial f_{5}}{\partial W}\right|=t_{1}<\infty$

$\left|\frac{\partial f_{5}}{\partial Y}\right|=0<\infty$,

$\left|\frac{\partial f_{5}}{\partial Z}\right|=\left|t_{2}\right|<\infty$,

$\left|\frac{\partial f_{5}}{\partial H}\right|=\left|-d_{1}-r_{1}-r_{2}\right|<\infty$.

TABLE 2: Notations and description of variables.

\begin{tabular}{lc}
\hline Variables & Descriptions \\
\hline$X(t)$ & Population size of susceptible prey \\
$W(t)$ & Population size of infected prey \\
$Y(t)$ & Population size of susceptible predator \\
$Z(t)$ & Population size of infected predator \\
$H(t)$ & Population size of infected population under treatment \\
\hline
\end{tabular}

(v) Positivity of $H(t)$ : from infected prey and infected predator population under treatment model in (8) $d$ $H / d t=t_{1} W+t_{2} Z-d_{1} H-r_{1} H-r_{2} H$, without loss of generality, after removing all positive terms, we have $d H / d t \geq-\left(d_{1}+r_{1}+r_{2}\right) H$ if and only if $d H / H$ $\geq-\left(d_{1}+r_{1}+r_{2}\right) d t$ integrating results, $\ln |H| \geq-\left(d_{1}\right.$ $\left.+r_{1}+r_{2}\right) t+Q$. Then, solving the variable $H$ yields $H \mid \geq e^{-\left(d_{1}+r_{1}+r_{2}\right) t+Q}$ which is an exponential function which is positive at all times. Therefore, $H(t)>0$, and hence, $H(t)$ is positive. Thus, variables $X(t), W$ $(t), Y(t), Z(t)$, and $H(t)$ are all positive quantities and remain in $\mathbb{R}_{+}^{5}$ for all $t$.

Theorem 3 (existence). All solutions of model (8) together with the initial conditions $X(0)>0, W(0) \geq 0, Y(0) \geq 0, Z(0)$ $\geq 0$, and $H(0) \geq 0$ exist in $\mathbb{R}_{+}^{5}$, i.e., the model variables $X(t)$, $W(t), Y(t), Z(t)$, and $H(t)$ exist for all $t$ and remain in $\mathbb{R}_{+}^{5}$.

Proof. Let the system of differential equation (8) be given as

$$
\begin{aligned}
& f_{1}=r X\left(1-\frac{X+W}{k}\right)+r_{1} H-\beta X W-\frac{p_{1} X Y}{s+X}-\frac{p_{3} X Z}{s+X} \\
& f_{2}=\beta X W-t_{1} W-d_{2} W-\frac{p_{2} W Y}{s+W}-\frac{p_{4} W Z}{s+W}, \\
& f_{3}=q \frac{p_{1} X Y}{s+X}+q \frac{p_{2} W Y}{s+W}+r_{2} H-\alpha Y Z-d_{3} Y, \\
& f_{4}=q \frac{p_{3} X Z}{s+X}+q \frac{p_{4} W Z}{s+W}+\alpha Y Z-t_{2} Z-d_{4} Z \\
& f_{5}=t_{1} W+t_{2} Z-d_{1} H-r_{1} H-r_{2} H .
\end{aligned}
$$

According to the Derrick and Groosman theorem, let $\Omega$ denote the region $\Omega=\left\{(X, W, \quad Y, Z, H) \in \mathbb{R}_{+}^{5} ; N \leq(\mu\right.$ $/ \lambda)\}$. Then, model (8) have a unique solution if $\left(\partial f_{i}\right) /\left(\partial x_{j}\right)$, $i, j=1,2,3,4,5$ are continuous and bounded in $\Omega$. Here, $x_{1}$ $=X, x_{2}=W, x_{3}=Y, x_{4}=Z$, and $x_{5}=H$. The continuity and the boundedness can be shown as follows.

Thus, all the partial derivatives in Table $1,\left(\partial f_{i}\right) /\left(\partial x_{j}\right), i$ $, j=1,2,3,4,5$ exist, continuous, and bounded in a region $\Omega$ for all positive values of model variables in Table 2 and 
TABLE 3: Notations and description of parameters.

\begin{tabular}{lc}
\hline Parameters & Description of parameters \\
\hline$r, k$ & Intrinsic growth rate and carrying capacity of susceptible prey \\
$\alpha, \beta$ & Disease transmission rates of prey and predator \\
$\mathrm{t}_{1}, \mathrm{t}_{2}$ & Treatment rate of infected prey and infected predator \\
$r_{1}, r_{2}$ & Recovery rate of infected prey and infected predator \\
$p_{i}, i=1,2,3,4$ & Predation coefficients \\
$f_{i}, i=1,2,3,4$ & Functional response \\
$d_{i}, i=1,2,3,4$ & Death rates \\
$\mathbf{q}, \mathbf{s}$ & Efficiency of predation and half-saturation constant \\
\hline
\end{tabular}

model parameters in Table 3. Hence, by the Derrick and Groosman theorem, a solution for the model (6)-(8) exists and is unique.

\section{Stability Analysis}

Stability analysis in the absence of predators in the model, that is, when $y(t)$ and $Z(t)$ are zero, model (8) can be written as

$$
\begin{aligned}
& \frac{d X}{d t}=r X\left(1-\frac{X+W}{k}\right)+r_{1} H-\beta X W=f(X, W, H), \\
& \frac{d W}{d t}=\beta X W-t_{1} W-d_{2} W=g(X, W, H), \\
& \frac{d H}{d t}=t_{1} W-d_{1} H-r_{1} H=h(X, W, H) .
\end{aligned}
$$

This system has trivial $E_{o}(0,0,0)$, axial $E_{A}$ $(k, 0,0)$, and positive $E_{o}(X, W, H)$ equilibrium points where

$$
\begin{aligned}
& X=k-\frac{k \beta}{r}-\frac{\beta}{d_{2}+t_{1}}+\frac{k \beta r_{1} t_{1}}{r\left(d_{1}+r_{1}\right)\left(d_{2}+t_{1}\right)}, \\
& W=\frac{\beta}{d_{2}+t_{1}}, \\
& H=\frac{\beta}{\left(d_{1}+r_{1}\right)\left(d_{2}+t_{1}\right)},
\end{aligned}
$$

with Jacobian matrix

$J(X, \quad W, \quad H)=\left(\begin{array}{ccc}r-\frac{2 r X}{k}-\frac{r W}{k}-\beta W & -\frac{r X}{k}-\beta X & r_{1} \\ \beta W & \beta X-t_{1}-d_{2} & 0 \\ 0 & t_{1} & -d_{1}-r_{1}\end{array}\right)$.

Theorem 4. The trivial equilibrium $E_{o}$ is a saddle point with unstable manifold in $X$-direction and stable manifold in the WY-plane.
Proof. The Jacobian matrix at $E_{o}$ is given by

$$
J\left(E_{o}\right)=\left(\begin{array}{ccc}
r & 0 & r_{1} \\
0 & -t_{1}-d_{2} & 0 \\
0 & t_{1} & -d_{1}-r_{1}
\end{array}\right)
$$

to compute eigenvalues compute the $\operatorname{det}\left(J\left(E_{o}\right)-\lambda I_{3}\right)=0$,

$$
\left|\begin{array}{ccc}
r-\lambda & 0 & r_{1} \\
0 & -t_{1}-d_{2}-\lambda & 0 \\
0 & t_{1} & -d_{1}-r_{1}-\lambda
\end{array}\right|=0
$$

Then, $(r-\lambda)\left(-t_{1}-d_{2}-\lambda\right)\left(-d_{1}-r_{1}-\lambda\right)=0$ is the characteristic polynomial.

Thus, eigenvalues are $\lambda_{1}=r>0, \lambda_{2}=-t_{1}-d_{2}<0, \lambda_{3}=-$ $d_{1}-r_{1}<0$ which is a saddle point with unstable manifold in the $X$-direction and stable manifold in the $W Y$-plane.

Theorem 5. The axial equilibrium $E_{A}$ is a saddle point if $\beta k$ $-t_{1}-d_{2}>0$ and unstable manifold in $X$-direction if $\beta k-t_{1}$ $-d_{2}<0$, then $E_{A}$ is stable.

Proof. The Jacobian matrix at $E_{A}$ is given by

$$
J\left(E_{A}\right)=\left(\begin{array}{ccc}
-r & -r-\beta k & r_{1} \\
0 & \beta k-t_{1}-d_{2} & 0 \\
0 & t_{1} & -d_{1}-r_{1}
\end{array}\right)
$$

to compute eigenvalues compute the $\operatorname{det}\left(J\left(E_{o}\right)-\lambda I_{3}\right)$ $=0$,

$$
\left|\begin{array}{ccc}
-r-\lambda & -r-\beta k & r_{1} \\
0 & \beta k-t_{1}-d_{2}-\lambda & 0 \\
0 & t_{1} & -d_{1}-r_{1}-\lambda
\end{array}\right|=0
$$

Then, $\quad(-r-\lambda)\left(\beta k-t_{1}-d_{2}-\lambda\right)\left(-d_{1}-r_{1}-\lambda\right)=0 \quad$ is characteristic polynomial. 
TABLE 4: Parameter value used in simulation.

\begin{tabular}{|c|c|c|}
\hline Name & Value & Description \\
\hline$r$ & 22.4000 & Growth rate of susceptible prey \\
\hline$k$ & $1.0000 E 03$ & Carrying capacity of susceptible prey \\
\hline$r^{-} 1$ & 1.0000 & Recovery rate of \\
\hline Beta & 2.4000 & Disease transmission rate in prey \\
\hline$P^{-} 1$ & 1.0000 & Predation coefficient of susceptible prey due to susceptible predator \\
\hline$s$ & 1.0000 & Half-saturated rate \\
\hline$P^{-} 3$ & 1.0000 & Predation coefficient of susceptible prey due to infected predator \\
\hline$t^{-} 1$ & 1.0000 & Treatment rate of infected prey \\
\hline$d^{-} 2$ & 1.0000 & Death rate of infected prey \\
\hline$P^{-} 2$ & 1.0000 & Predation coefficient of infected prey due to predators \\
\hline$P^{-} 4$ & 1.0000 & Predation rate of infected prey due to infected predator \\
\hline$q$ & 1.0000 & Efficiency of predation \\
\hline$r^{-} 2$ & 1.0000 & Recovery rate of susceptible predator \\
\hline Alpha & 2.6000 & Disease transmission rate in predator \\
\hline$d^{-} 3$ & 1.0000 & Death rate of susceptible predator \\
\hline$t^{-} 2$ & 1.0000 & Treatment rate of infected predator \\
\hline$d^{-} 4$ & 1.0000 & Death rate of infected predator \\
\hline$d^{-} 1$ & 1.0000 & Death rate of both infected and infected predator under treatment \\
\hline
\end{tabular}

Thus, $\quad \lambda_{1}=-r<0, \lambda_{2}=\beta k-t_{1}-d_{2}, \lambda_{3}=-d_{1}-r_{1}<0$; hence, the axial equilibrium point is the saddle point if $\beta k$ $-t_{1}-d_{2}>0$ and stable if $\beta k-t_{1}-d_{2}<0$.

Stability analysis in the absence of infectious disease in system (8), that is, when there is no disease, $W(t), Z(t)$, and $H(t)$ are all zero and model (8) becomes

$$
\begin{aligned}
& \frac{d X}{d t}=r X\left(1-\frac{X+W}{k}\right)-\frac{p_{1} X Y}{s+X}=f(X, Y), \\
& \frac{d Y}{d t}=q \frac{p_{1} X Y}{s+X}-d_{3} Y=g(X, Y) .
\end{aligned}
$$

This system contains trivial $E_{o}\left(\begin{array}{ll}0, & 0\end{array}\right)$, axial $E_{A}(k, 0)$, and positive $E_{o}(X, Y)$ equilibrium points, where

$X=\frac{r(k-s)+\sqrt{r} \sqrt{k^{2} r+2 k r s+r X s^{2}-4 k p_{1}}}{2 r}$,

$Y=\frac{q p_{1}}{d_{3}\left(s+\left(r(k-s)+\sqrt{r} \sqrt{k^{2} r+2 k r s+r s^{2}-4 k p_{1}}\right) / 2 r\right)}$,
TABLE 5: Initial conditions used for model variables.

\begin{tabular}{lcc}
\hline Name & Value & Description \\
\hline$X[t 0]$ & $1.2000 E 04$ & Initial \# susceptible prey \\
$W[t 0]$ & 200.0000 & Initial \# infected prey \\
$H[t 0]$ & 1.0000 & Initial \# under treated prey predator \\
$Y[t 0]$ & 160.0000 & Initial \# susceptible predator \\
$Z[t 0]$ & 180.0000 & Initial \# of infected predator \\
\hline
\end{tabular}

and Jacobian matrix is given by

$$
J(X, Y)=\left(\begin{array}{cc}
r-\frac{2 r X}{k}-\frac{p_{1} Y S}{(s+X)^{2}} & \frac{q p_{1} X}{s+k} \\
\frac{q p_{1} Y S}{(s+X)^{2}} & \frac{q p_{1} X}{s+X}-d_{3}
\end{array}\right) .
$$

Theorem 6. The trivial equilibrium $E_{o}$ is a saddle point with unstable manifold in the X-direction and stable manifold in the $Y$-direction.

Proof. The Jacobian matrix at $E_{o}$ is given by

$$
J\left(E_{o}\right)=\left(\begin{array}{cc}
r & 0 \\
0 & -d_{3}
\end{array}\right)
$$

hence, eigenvalues are $\lambda_{1}=r>0, \lambda_{2}=-d_{3}<0$ which is a saddle point. 


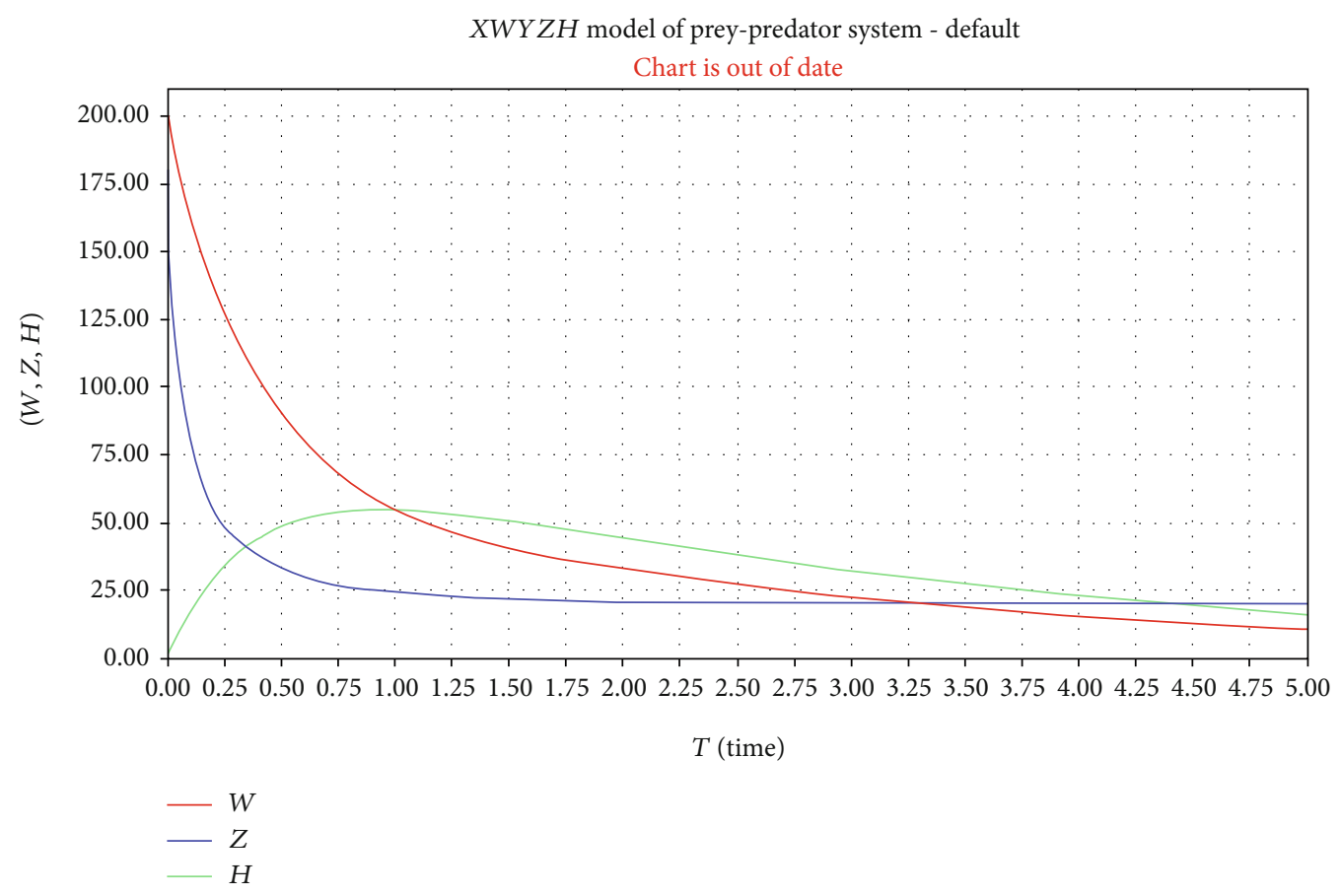

FIGURE 2: Infected prey-predator with treatment.

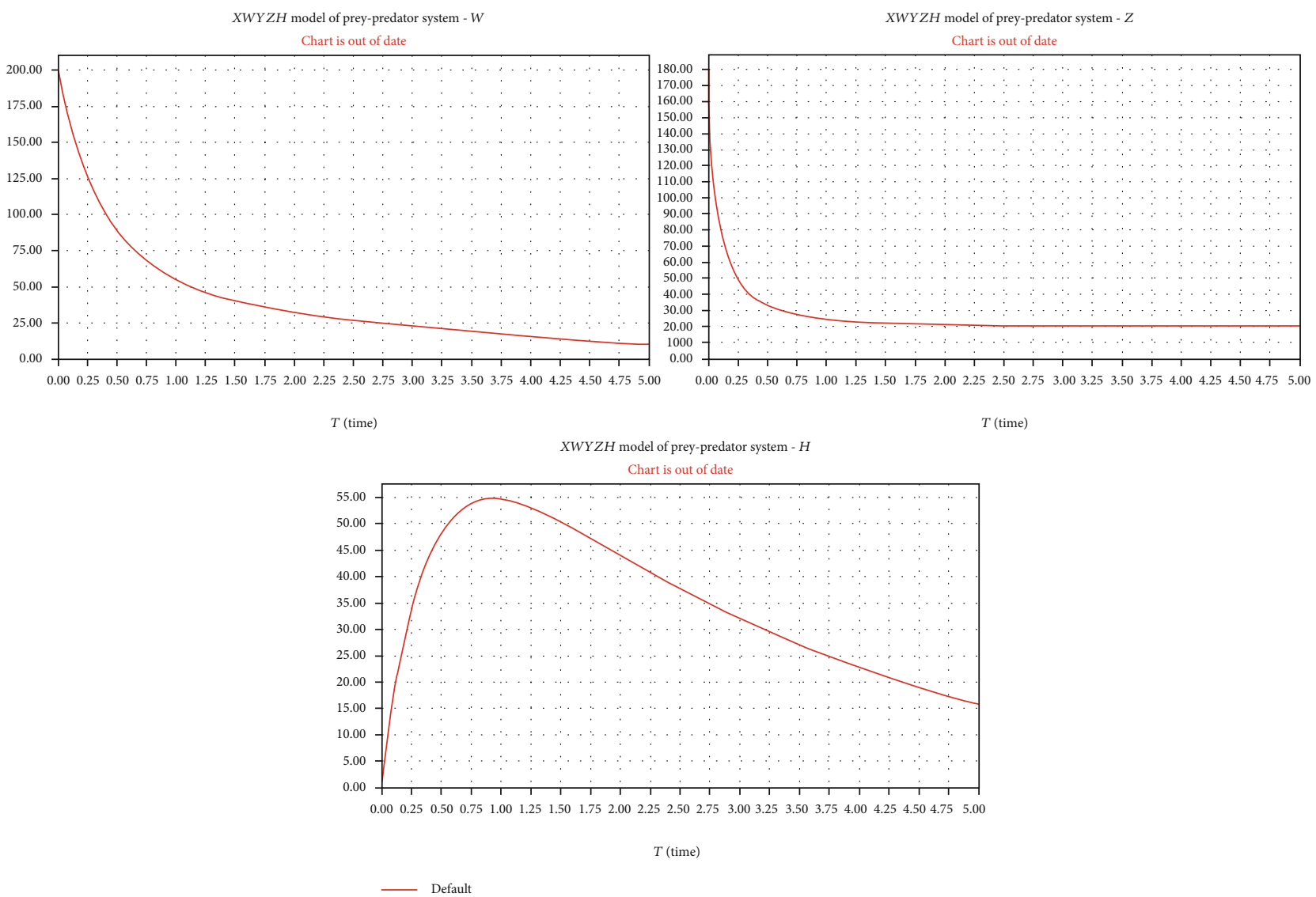

Figure 3: Plots for $W, Z$, and $H$. 


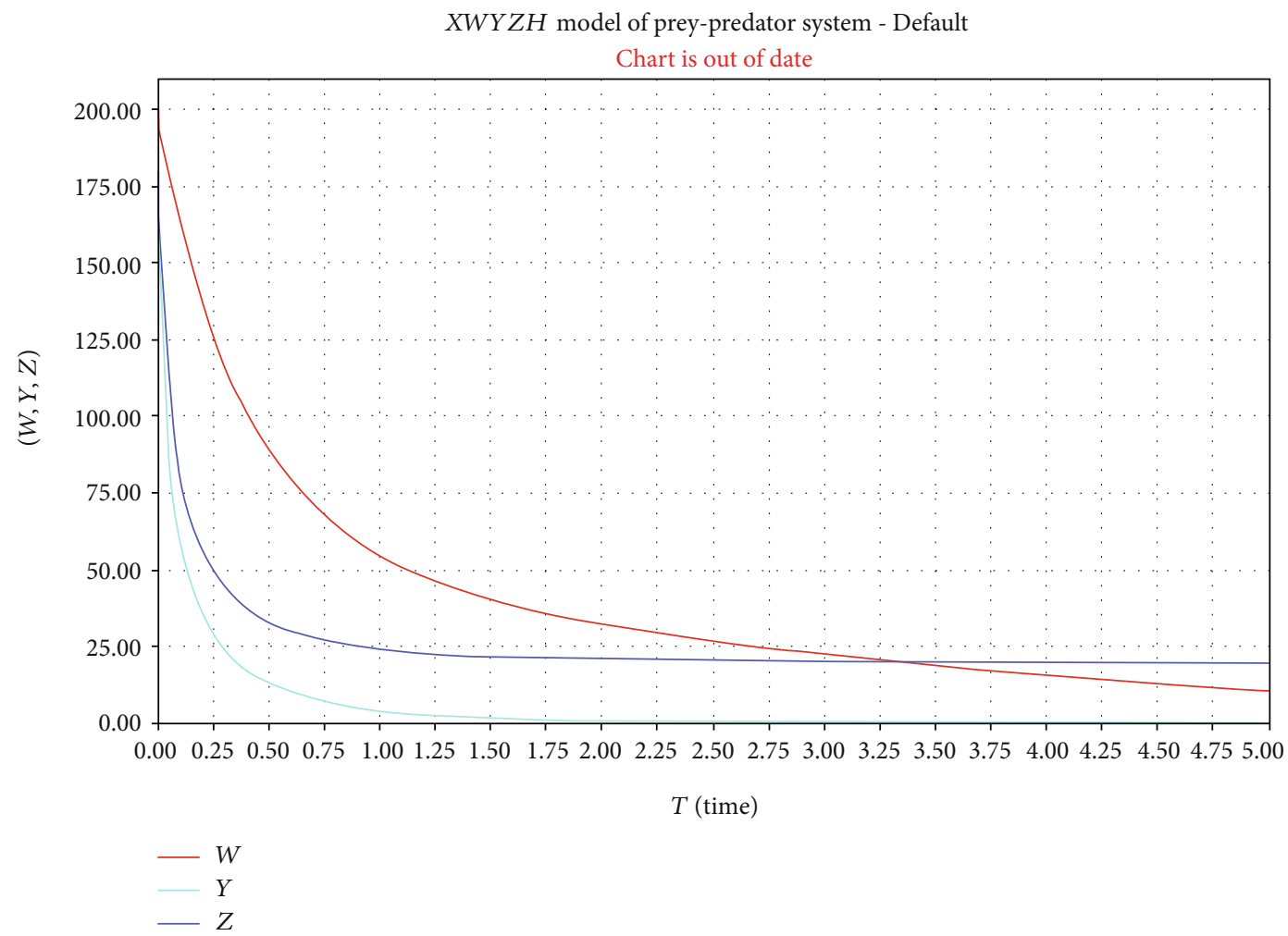

Figure 4: High infection and predation.
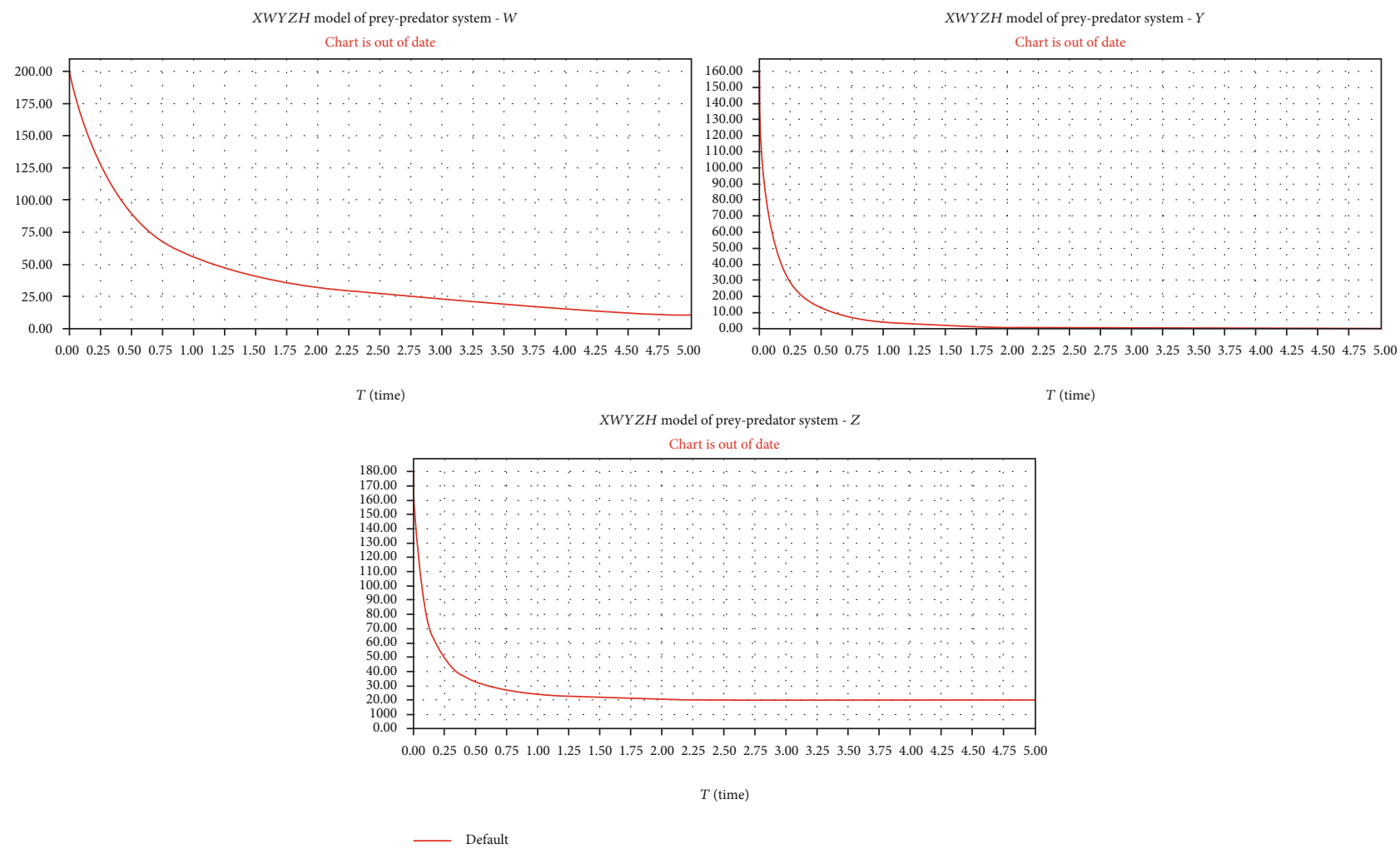

Figure 5: Individual plot for $W, Y$, and $Z$. 
Theorem 7. The axial equilibrium $E_{A}$ is stable if $q p_{1} k /(s+k)$ $-d_{3}<0$, otherwise unstable.

Proof. The Jacobian matrix at $E_{A}$ is given by

$$
J\left(E_{A}\right)=\left(\begin{array}{cc}
-r & -\frac{p_{1} k}{s+k} \\
0 & -\frac{p_{1} k}{s+k}-d_{3}
\end{array}\right) .
$$

To find eigenvalues, compute $\operatorname{det}\left(J\left(E_{A}\right)-\lambda I_{3}\right)=0$,

$$
\left|\begin{array}{cc}
-r-\lambda & -\frac{p_{1} k}{s+k} \\
0 & \frac{q p_{1} k}{s+k}-d_{3}-\lambda
\end{array}\right|=0 .
$$

Eigen values are $\lambda_{1}=-r<0, \lambda_{2}=q p_{1} k /(s+k)-d_{3}$.

Thus, $E_{A}$ is stable if $q p_{1} k /(s+k)-d_{3}<0$ and otherwise unstable.

Theorem 8. The positive equilibrium $E$ is stable if $[r-2 r X / k$ $\left.-p_{1} Y S /(s+X)^{2}\right]+\left[q p_{1} k /(s+k)-d_{3}\right]>0$ and $[r-2 r X / k-$ $\left.p_{1} Y S /(s+X)^{2}\right]\left[q p_{1} k /(s+k)-d_{3}\right]+q p_{1}{ }^{2} X Y S /(s+X)^{3}>0$.

Proof. The Jacobian matrix at $E$ is given by

$$
J(X, Y)=\left(\begin{array}{cc}
r-\frac{2 r X}{k}-\frac{p_{1} Y S}{(s+X)^{2}} & \frac{q p_{1} X}{s+k} \\
\frac{q p_{1} Y S}{(s+X)^{2}} & \frac{q p_{1} X}{s+X}-d_{3}
\end{array}\right)
$$

Then, compute det $\left(J(E)-\lambda I_{3}\right)=0$,

$$
\left|\begin{array}{cc}
r-\frac{2 r X}{k}-\frac{p_{1} Y S}{(s+X)^{2}}-\lambda & \frac{q p_{1} X}{s+k} \\
\frac{q p_{1} Y S}{(s+X)^{2}} & \frac{q p_{1} X}{s+X}-d_{3}-\lambda
\end{array}\right|=0 .
$$

Then,

$$
(\underbrace{r-\frac{2 r X}{k}-\frac{p_{1} Y S}{(s+X)^{2}}}_{a}-\lambda)(\underbrace{\frac{q p_{1} X}{s+X}-d_{3}}_{b}-\lambda)+\underbrace{\frac{s q p_{1}{ }^{2} X Y}{(s+X)^{3}}}_{c}=0,
$$

is a characteristic polynomial.
Using the Routh-Hurwitz criterion, the quadratic polynomial is stable if $a+b>0, a b+c>0$, otherwise unstable.

Equilibrium points in model (8) are steady state points of the form $(X, W, Y, Z, H)$ of model $((8))$ that satisfies $d X / d t=d W / d t=d Y / d t=d Z / d t=d H / d t=0$, provided that each variable is nonnegative. In model $((8))$, five steady state points are identified and listed here: trivial steady state $E_{o}(0,0,0,0,0)$, axial steady state $E_{A}$ $(k, \quad 0, \quad 0, \quad 0,0)$, disease-free steady state $\bar{E}$ $(\bar{X}, 0, \bar{Y}, 0,0)$, and endemic steady state $E^{*}\left(X^{*}\right.$, $\left.W^{*}, Y^{*}, Z^{*}, H^{*}\right)$. Computation of disease free and endemic equilibrium points is presented as follows.

Disease-free equilibrium points (DFEP) of model (8) are steady state solutions when there is no infectious disease in the population. In the absence of infectious disease in the prey-predator system, the variables $W(t)=Z(t)=H(t)=0$ and $d X / d t=d W / d t=d Y / d t=d Z / d t=d H / d t=0$. Then, model (8) become

$$
\left\{\begin{array}{l}
r \bar{X}\left(1-\frac{\bar{X}}{k}\right)-\frac{p_{1} \bar{X} \bar{Y}}{s+X}=0 \\
\frac{q p_{1} \bar{X} \bar{Y}}{s+\bar{X}}-d_{3} \bar{Y}=0
\end{array}\right.
$$

Thus, solving $\bar{X}$ and $\bar{Y}$, it is found that $\bar{X}=d_{3} s /\left(q p_{1}-\right.$ $\left.d_{3}\right)$ and $\bar{Y}=r s q\left(k q p_{1}-k d_{3}-d_{3} s\right) /\left(q p_{1}-d_{3}\right)^{2} k$, and hence, disease-free equilibrium point (DFEP) of model (8) is given by

$$
\begin{aligned}
& \bar{E}=\left\{\begin{array}{lllll}
\bar{X}, & 0, & \bar{Y}, & 0, & 0
\end{array}\right\} \\
& =\left\{\frac{d_{3} s}{q p_{1}-d_{3}}, \quad 0, \frac{r s q\left(k q p_{1}-k d_{3}-d_{3} s\right)}{\left(q p_{1}-d_{3}\right)^{2} k}, 0, \quad 0\right\} .
\end{aligned}
$$

The endemic equilibrium point (EEP) is positive equilibrium point $E^{*}\left(X^{*}, W^{*}, Y^{*}, Z^{*}, H^{*}\right)$ obtained by solving model equation (8) as $d X / d t=d W / d t=d Y / d t=d Z / d t=d$ $H / d t=0$ for which all variables are nonzero

$$
\left\{\begin{array}{l}
r X^{*}\left(1-\frac{X^{*}+W^{*}}{k}\right)+r_{1} H^{*}-\beta X^{*} W^{*}-\frac{p_{1} X^{*} Y^{*}+p_{3} X^{*} Z^{*}}{s+X^{*}}=0, \\
\beta X^{*} W^{*}-t_{1} W^{*}-d_{2} W^{*}-\frac{p_{2} W^{*} Y^{*}+p_{4} W^{*} Z^{*}}{s+W^{*}}=0, \\
q \frac{p_{1} X^{*} Y^{*}}{s+X^{*}}+q \frac{p_{2} W^{*} Y^{*}}{s+W^{*}}+r_{2} H^{*}-\alpha Y^{*} Z^{*}-d_{3} Y^{*}=0, \\
q \frac{p_{3} X^{*} Z^{*}}{s+X^{*}}+q \frac{p_{4} W^{*} Z^{*}}{s+W^{*}}+\alpha Y^{*} Z^{*}-\left(t_{2}+d_{4}\right) Z^{*}=0, \\
t_{1} W^{*}+t_{2} Z^{*}-\left(d_{1}+r_{1}+r_{2}\right) H^{*}=0 .
\end{array}\right.
$$

Then, solving for the variables $X^{*}, W^{*}, Y^{*}, Z^{*}$, and $H^{*}$, the endemic equilibrium points of the model exists and a simplified result is obtained 


$$
\begin{aligned}
X^{*} & =\left(-r \beta d_{1}+[2 k r-r s-2 k \beta] d_{1} d_{2}-r \beta r_{1}+2 k r d_{2} r_{1}\right), \\
W^{*} & =\frac{\beta-s\left(d_{2}+t_{1}\right)-\sqrt{4\left(s \beta-p_{2}-p_{4}\right)\left(t_{1}+d_{2}\right)+\left(s\left[t_{1}+d_{2}\right]-\beta\right)^{2}}}{2\left(t_{1}+d_{2}\right)}, \\
Y^{*} & =\frac{1}{d_{3}}\left(-\alpha-\frac{r r_{2}}{r_{1}}+\frac{r \beta r_{2}}{r_{1}}+\frac{r \beta r_{2}}{2 k r_{1}\left(t_{1}+d_{2}\right)}-\frac{r s d_{2} r_{2}}{2 k r_{1}\left(t_{1}+d_{2}\right)}-\frac{r s r_{2} t_{1}}{2 k r_{1}\left(t_{1}+d_{2}\right)}\right), \\
Z^{*} & =\frac{1}{t_{2}+d_{4}}\left(\begin{array}{c}
\left.\alpha+\frac{q p_{4}}{s+\left(\beta-\left(t_{1}+d_{2}\right)+\sqrt{4\left(s \beta-p_{2}-p_{4}\right)\left(t_{1}+d_{2}\right)+\left(s\left[t_{1}+d_{2}\right]-\beta\right)^{2}}\right) / 2\left(t_{1}+d_{2}\right)}\right)
\end{array}\right) \\
H^{*} & =\frac{1}{r_{1}}\left(\beta-r+\frac{r \beta-r s d_{2}-r s t_{1}}{2 k\left(t_{1}+d_{2}\right)}\right) .
\end{aligned}
$$

To study the stability analysis of equilibrium points of model (8), it is better to linearize model (8) using variation matrix. Let us represent the model (8) as system of parametrical equations as follows:

$$
\begin{aligned}
& \frac{d X}{d t}=f(X, W, Y, Z, H)=r X\left(1-\frac{X+W}{k}\right) \\
&+r_{1} H-\beta X W-\frac{p_{1} X Y}{s+X}-\frac{p_{3} X Z}{s+X}, \\
& \frac{d W}{d t}= g(X, W, Y, Z, H)=\beta X W-t_{1} W \\
&-d_{2} W-\frac{p_{2} W Y}{s+W}-\frac{p_{4} W Z}{s+W}, \\
& \frac{d Y}{d t}= h(X, W, Y, Z, H)=q \frac{p_{1} X Y}{s+X} \\
&+q \frac{p_{2} W Y}{s+W}+r_{2} H-\alpha Y Z-d_{3} Y, \\
& \frac{d Z}{d t}= i(X, W, Y, Z, H)=q \frac{p_{3} X Z}{s+X} \\
&+q \frac{p_{4} W Z}{s+W}+\alpha Y Z-t_{2} Z-d_{4} Z,
\end{aligned}
$$

$\frac{d H}{d t}=j(X, W, Y, Z, H)=t_{1} W+t_{2} Z-d_{1} H-r_{1} H-r_{2} H$. by

Then, the variation matrix of these functions (36) is given

$$
V(X, W, Y, Z, H)=\left(\begin{array}{ccccc}
f_{X} & f_{W} & f_{Y} & f_{Z} & f_{H} \\
g_{X} & g_{W} & g_{Y} & g_{Z} & g_{H} \\
h_{X} & h_{W} & h_{Y} & h_{Z} & h_{H} \\
i_{X} & i_{W} & i_{Y} & i_{Z} & i_{H} \\
j_{X} & j_{W} & j_{Y} & j_{Z} & j_{H}
\end{array}\right),
$$

where each element of the matrix represents partial derivatives of functions (36) with respect to model variables and computations of each element of the variation matrix are given as

$V(X, W, Y, Z, H)=\left(\begin{array}{ccccc}f_{X} & -\frac{r X}{k}-\beta X & -\frac{p_{1} X}{s+X} & -\frac{p_{3} X}{s+X} & r_{1} \\ \beta W & g_{W} & -\frac{p_{2} W}{s+W} & -\frac{p_{4} W}{s+w} & 0 \\ \frac{s q p_{1} Y}{(s+X)^{2}} & \frac{s q p_{2} Y}{(s+X)^{2}} & h_{Y} & -\alpha y-d_{3} & r_{2} \\ \frac{s q p_{3} Z}{(s+X)^{2}} & \frac{s q p_{4} Z}{(s+W)^{2}} & \alpha Z & i_{z} & 0 \\ 0 & t_{1} & 0 & t_{2} & -d_{1}-r_{1}-r_{2}\end{array}\right)$,

where, $f_{X}=r-2 r x / k-r W / k-\beta W-s\left(p_{1} Y+p_{3} Z\right) /(s+X)^{2}$, $g_{W}=\beta X-t_{1}-d_{2}-s\left(p_{2} Y+p_{4} Z\right) /(s+W)^{2}, h_{Y}=q p_{1} X /(s+$ $X)+q p_{2} W /(s+W)-\alpha Z-d_{3}, i_{z}=q p_{3} X /(s+X)+q p_{4} W /(s$ $+W)-\alpha Y-t_{2}-d_{4}$.

Theorem 9 (TEP). Trivial equilibrium point $E_{o}$

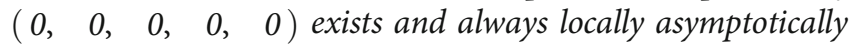
unstable.

Proof. Consider the variation matrix (40) at $E_{o}$.

$$
V\left(E_{o}\right)=\left(\begin{array}{ccccc}
r & 0 & 0 & 0 & r_{1} \\
0 & -t_{1}-d_{2} & 0 & 0 & 0 \\
0 & 0 & -d_{3} & 0 & r_{2} \\
0 & 0 & 0 & -t_{2}-d_{4} & 0 \\
0 & t_{1} & 0 & t_{2} & -d_{1}-r_{1}-r_{2}
\end{array}\right) .
$$

Eigenvalue of variation matrix can be computed from the characteristic polynomial det $\left(V\left(E_{o}\right)-\lambda I_{5}\right)=0$ 
$\left|\begin{array}{ccccc}r-\lambda & 0 & 0 & 0 & r_{1} \\ 0 & -t_{1}-d_{2}-\lambda & 0 & 0 & 0 \\ 0 & 0 & -d_{3}-\lambda & 0 & r_{2} \\ 0 & 0 & 0 & -t_{2}-d_{4}-\lambda & 0 \\ 0 & a_{1} & 0 & a_{2} & -d_{1}-r_{1}-r_{2}-\lambda\end{array}\right|=0$

$\Longrightarrow(r-\lambda)\left(-t_{1}-d_{2}-\lambda\right)\left(-d_{3}-\lambda\right)\left(-t_{2}-d_{4}-\lambda\right)\left(-d_{1}-\right.$ $\left.r_{1}-r_{2}-\lambda\right)=0$ is the characteristic polynomial. The eigen values are $\lambda_{1}=r, \lambda_{2}=-t_{1}-d_{2}, \lambda_{3}=-d_{3}, \lambda_{4}=-t_{2}-d_{4}, \lambda_{5}=$ $-d_{1}-r_{1}-r_{2}$. Thus, the trivial equilibrium point is locally asymptotically unstable due to the parameter $r$ is nonnegative.

Theorem 10 (AEP). Axial equilibrium point $E_{A}(k, 0,0,0,0)$ exists and always locally asymptotically stable in model (8) if and only if model parameters satisfy the conditions: $\beta k-t_{1}$ $-d_{2}<0, q p_{1} k /(s+k)-d_{3}<0$, and $q p_{3} k /(s+k)-t_{2}-d_{4}<0$. Otherwise, $E_{A}$ is locally asymptotically unstable.

Proof. Consider the variation matrix $(40)$ at $J\left(E_{A}\right)$

$$
V\left(E_{A}\right)=\left(\begin{array}{ccccc}
-r & -r-\beta k & -\frac{p_{1} k}{s+k} & -\frac{p_{3} k}{s+k} & r_{1} \\
0 & \beta k-t_{1}-d_{2} & 0 & 0 & 0 \\
0 & 0 & \frac{q p_{1} k}{s+k}-d_{3} & d_{3} & r_{2} \\
0 & 0 & 0 & \frac{q p_{3} k}{s+k}-t_{2}-d_{4} & 0 \\
0 & t_{1} & 0 & t_{2} & -d_{1}-r_{1}-r_{2}
\end{array}\right)
$$

Then, it is possible to find eigenvalue of this variation matrix as $\operatorname{det}\left(V\left(E_{A}-\lambda I_{5}\right)=0\right.$,

$$
\left|\begin{array}{ccccc}
-r-\lambda & -r-\beta k & -\frac{p_{1} k}{s+k} & -\frac{p_{3} k}{s+k} & r_{1} \\
0 & \beta k-t_{1}-d_{2}-\lambda & 0 & 0 & 0 \\
0 & 0 & \frac{q p_{1} k}{s+k}-d_{3}-\lambda & d_{3} & r_{2} \\
0 & 0 & 0 & \frac{q p_{3} k}{s+k}-t_{2}-d_{4}-\lambda & 0 \\
0 & t_{1} & 0 & t_{2} & -d_{1}-r_{1}-r_{2}-\lambda
\end{array}\right|=0
$$

Use first column to find the determinant,

$$
\Longrightarrow(-r-\lambda)\left|\begin{array}{cccc}
\beta k-t_{1}-d_{2}-\lambda & 0 & 0 & 0 \\
0 & \frac{q p_{1} k}{s+k}-d_{3}-\lambda & d_{3} & r_{2} \\
0 & 0 & \frac{q p_{3} k}{s+k}-t_{2}-d_{4}-\lambda & 0 \\
t_{1} & 0 & t_{2} & -d_{1}-r_{1}-r_{2}-\lambda
\end{array}\right|=0 .
$$


Use first row to compute the remaining determinant,

$$
\Longrightarrow(-r-\lambda)\left(\beta k-t_{1}-d_{2}-\lambda\right)\left|\begin{array}{ccc}
\frac{q p_{1} k}{s+k}-d_{3}-\lambda & d_{3} & r_{2} \\
0 & \frac{q p_{3} k}{s+k}-t_{2}-d_{4}-\lambda & 0 \\
0 & t_{2} & -d_{1}-r_{1}-r_{2}-\lambda
\end{array}\right|=0
$$

Use first column to get the characteristic polynomial,

$$
\begin{gathered}
\Longrightarrow(-r-\lambda)\left(\beta k-t_{1}-d_{2}-\lambda\right)\left(\frac{q p_{1} k}{s+k}-d_{3}-\lambda\right) \\
\cdot\left|\begin{array}{cc}
\frac{q p_{3} k}{s+k}-t_{2}-d_{4}-\lambda & 0 \\
t_{2} & -d_{1}-r_{1}-r_{2}-\lambda
\end{array}\right|=0, \\
\Longrightarrow(-r-\lambda)\left(\beta k-t_{1}-d_{2}-\lambda\right)\left(\frac{q p_{1} k}{s+k}-d_{3}-\lambda\right) \\
\cdot\left(\frac{q p_{3} k}{s+k}-t_{2}-d_{4}-\lambda\right)\left(-d_{1}-r_{1}-r_{2}-\lambda\right)=0,
\end{gathered}
$$

is characteristic polynomial. Then, eigenvalues are

$$
\begin{aligned}
\lambda_{1} & =-r, \lambda_{2}=-d_{1}-r_{1}-r_{2}, \lambda_{3}=\beta k-t_{1}-d_{2}, \lambda_{4} \\
& =\frac{q p_{1} k}{s+k}-d_{3}, \lambda_{5}=\frac{q p_{3} k}{s+k}-t_{2}-d_{4} .
\end{aligned}
$$

The axial equilibrium point $E_{A}$ is locally asymptotically stable, if

$$
\beta k-t_{1}-d_{2}<0, \frac{q p_{1} k}{s+k}-d_{3}<0, \frac{q p_{3} k}{s+k}-t_{2}-d_{4}<0 .
$$

Otherwise, $E_{A}$ is locally asymptotically unstable.

Theorem 11 (DFEP). The disease-free equilibrium point $\bar{E}$ $(\bar{X}, \quad 0, \quad \bar{Y}, \quad 0, \quad 0)=\left\{d_{3} s /\left(q p_{1}-d_{3}\right), \quad 0, \quad r s q\left(k q p_{1} \quad-\right.\right.$ $\left.\left.k d_{3}-d_{3} s\right) /\left(q p_{1}-d_{3}\right)^{2} k, 0,0\right\}$ exists and is always locally asymptotically stable if and only if the model parameter satisfy conditions (i) $\beta \bar{X}-t_{1}-d_{2}-s p_{2} \bar{Y} / s^{2} \leq 0$, (ii) $q p_{3} \bar{X} /(s+X)-$ $\alpha \bar{Y}-t_{2}-d_{4} \leq 0$, (iii) $\left\{r-2 r \bar{X} / k-s p_{1} \bar{Y} /(s+\bar{X})^{2}\right\}+\left\{q p_{1} \bar{X} /(\right.$ $\left.s+\bar{X})-d_{3}\right\}>0$, and (iv) $\left\{r-2 r \bar{X} / k-s p_{1} \bar{Y} /(s+\bar{X})^{2}\right\}\left\{q p_{1} \bar{X} /\right.$ $\left.(s+\bar{X})-d_{3}\right\}+s q p_{1}{ }^{2} \bar{X}^{2} /(s+\bar{X})^{3}>0$.

Proof. Consider the variation matrix (40) at disease-free equilibrium point $\bar{E}(\bar{X}, 0, \overline{Y,}, 0,0)$ such that

$$
V(\bar{E})=\left(\begin{array}{ccccc}
r-\frac{2 r \bar{X}}{k}-\frac{s p_{1} \bar{Y}}{(s+\bar{X})^{2}} & -\frac{r \bar{X}}{k}-\beta \bar{X} & -\frac{p_{1} \bar{X}}{s+\bar{X}} & -\frac{p_{3} \bar{X}}{s+\bar{X}} & r_{1} \\
0 & \beta \bar{X}-t_{1}-d_{2}-\frac{s p_{2} \bar{Y}}{s^{2}} & 0 & 0 & 0 \\
\frac{s q p_{1} \bar{X}}{(s+\bar{X})^{2}} & \frac{s q p_{2} \bar{Y}}{(s+\bar{X})^{2}} & \frac{q p_{1} \bar{X}}{s+\bar{X}}-d_{3} & -\alpha \bar{Y}-d_{3} & r_{2} \\
0 & 0 & 0 & \frac{q p_{3} \bar{X}}{s+\bar{X}}-\alpha \bar{Y}-t_{2}-d_{4} & 0 \\
0 & t_{1} & 0 & t_{2} & -d_{1}-r_{1}-r_{2}
\end{array}\right)
$$


Then, it is possible to find the determinant of the variation matrix as $\operatorname{det}\left(V(\bar{E})-\lambda I_{5}\right)$

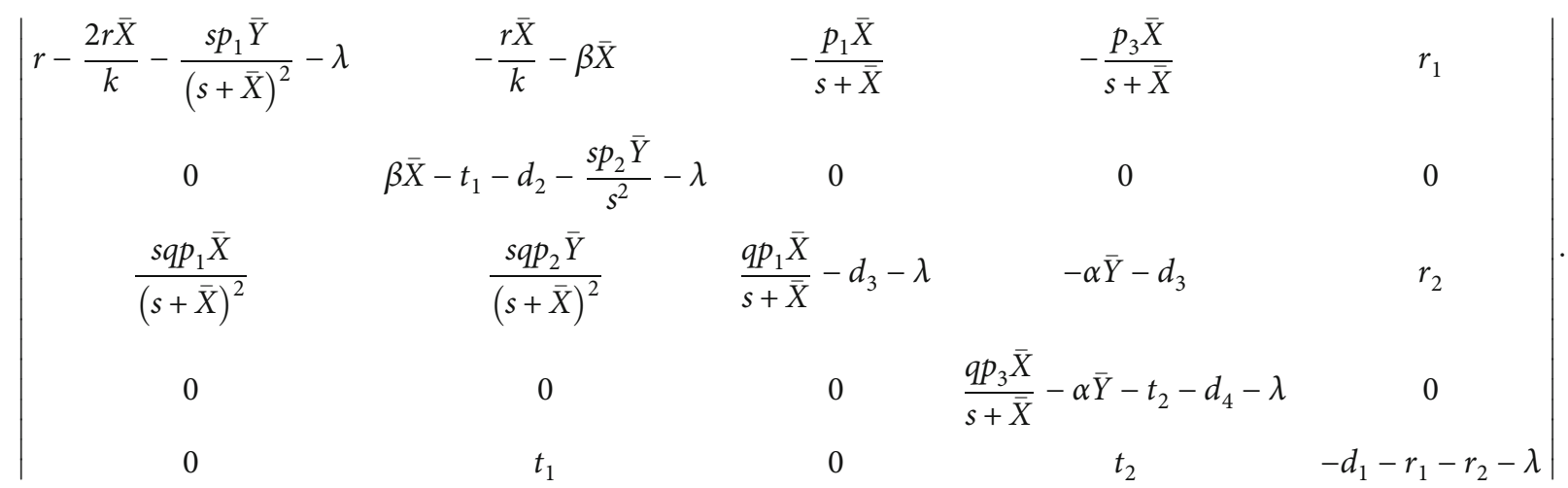

To find eigenvalues, compute the determinant using second row

$$
\left(\beta \bar{X}-t_{1}-d_{2}-\frac{s p_{2} \bar{Y}}{s^{2}}-\lambda\right) *\left|\begin{array}{cccc}
r-\frac{2 r \bar{X}}{k}-\frac{s p_{1} \bar{Y}}{(s+\bar{X})^{2}}-\lambda & -\frac{p_{1} \bar{X}}{s+\bar{X}} & -\frac{p_{3} \bar{X}}{s+\bar{X}} & r_{1} \\
\frac{s q p_{1} \bar{X}}{(s+\bar{X})^{2}} & \frac{q p_{1} \bar{X}}{s+\bar{X}}-d_{3}-\lambda & -\alpha Y-d_{3} & r_{2} \\
0 & 0 & \frac{q p_{3} \bar{X}}{s+\bar{X}}-\alpha Y-t_{2}-d_{4}-\lambda & 0 \\
0 & 0 & 0 & -d_{1}-r_{1}-r_{2}-\lambda
\end{array}\right| .
$$

Now again use third row to find determinant:

$$
\begin{aligned}
& \left(\beta \bar{X}-t_{1}-d_{2}-\frac{s p_{2} \bar{Y}}{s^{2}}-\lambda\right)\left(\frac{q p_{3} \bar{X}}{s+\bar{X}}-\alpha \bar{Y}-t_{2}-d_{4}-\lambda\right) \\
& *\left|\begin{array}{ccc}
r-\frac{2 r \bar{X}}{k}-\frac{s p_{1} \bar{Y}}{(s+\bar{X})^{2}}-\lambda & -\frac{p_{1} \bar{X}}{s+\bar{X}} & r_{1} \\
\frac{s q p_{1} \bar{X}}{(s+\bar{X})^{2}} & \frac{q p_{1} \bar{X}}{s+\bar{X}}-d_{3}-\lambda & r_{2} \\
0 & 0 & -d_{1}-r_{1}-r_{2}-\lambda
\end{array}\right|
\end{aligned}
$$

Then, use third row to find characteristic polynomial

$$
\begin{aligned}
& \left(\beta \bar{X}-t_{1}-d_{2}-\frac{s p_{2} \bar{Y}}{s^{2}}-\lambda\right) \\
& \cdot\left(\frac{q p_{3} \bar{X}}{s+\bar{X}}-\alpha \bar{Y}-t_{2}-d_{4}-\lambda\right)\left(-d_{1}-r_{1}-r_{2}-\lambda\right) \\
& \text { *|cc} \begin{array}{cc}
r-\frac{2 r \bar{X}}{k}-\frac{s p_{1} \bar{Y}}{(s+\bar{X})^{2}}-\lambda & -\frac{p_{1} \bar{X}}{s+X} \\
\frac{s q p_{1} \bar{X}}{(s+\bar{X})^{2}} & \frac{q p_{1} \bar{X}}{s+\bar{X}}-d_{3}-\lambda
\end{array} \mid
\end{aligned}
$$




$$
\begin{aligned}
& \cdot\left(\beta \bar{X}-t_{1}-d_{2}-\frac{s p_{2} \bar{Y}}{s^{2}}-\lambda\right)\left(\frac{q p_{3} \bar{X}}{s+X}-\alpha \bar{Y}-t_{2}-d_{4}-\lambda\right) \\
& \cdot\left(-d_{1}-r_{1}-r_{2}-\lambda\right) *\left\{\left(r-\frac{2 r \bar{X}}{k}-\frac{s p_{1} \bar{Y}}{(s+\bar{X})^{2}}-\lambda\right)\right. \\
& \left.\cdot\left(\frac{q p_{1} \bar{X}}{s+\bar{X}}-d_{3}-\lambda\right)+\frac{s q p_{1}{ }^{2} \bar{X}^{2}}{(s+\bar{X})^{3}}\right\}=0 .
\end{aligned}
$$

Eigenvalues are

$$
\begin{aligned}
\lambda_{1}= & \beta \bar{X}-t_{1}-d_{2}-\frac{s p_{2} \bar{Y}}{s^{2}}, \lambda_{2}=\frac{q p_{3} \bar{X}}{s+\bar{X}} \\
& -\alpha \bar{Y}-t_{2}-d_{4}, \lambda_{3}=-d_{1}-r_{1}-r_{2},
\end{aligned}
$$

and the remaining eigenvalues can be obtained from the roots of quadratic equation:

$$
(\underbrace{r-\frac{2 r \bar{X}}{k}-\frac{s p_{1} \bar{Y}}{(s+\bar{X})^{2}}}_{a}-\lambda)(\underbrace{\frac{q p_{1} \bar{X}}{s+\bar{X}}-d_{3}}_{b}-\lambda)+\underbrace{\frac{s q p_{1}^{2} \bar{X}^{2}}{(s+\bar{X})^{3}}}_{c}=0 .
$$

It is known that a quadratic equation $(a-\lambda)(b-\lambda)+c$ $=0$ is locally asymptotically stable if and only if $a+b>0$ and $a b+c>0$, using such Routh-Hurwitz criterion, the disease-free equilibrium point $\bar{E}(\bar{X}, 0, \overline{Y, 0}, 0)$ is locally asymptotically stable if

$$
\begin{aligned}
& \left\{r-\frac{2 r \bar{X}}{k}-\frac{s p_{1} \bar{Y}}{(s+\bar{X})^{2}}\right\}+\left\{\frac{q p_{1} \bar{X}}{s+\bar{X}}-d_{3}\right\} \\
& >0,\left\{r-\frac{2 r \bar{X}}{k}-\frac{s p_{1} \bar{Y}}{(s+\bar{X})^{2}}\right\}\left\{\frac{q p_{1} \bar{X}}{s+\bar{X}}-d_{3}\right\}+\frac{s q p_{1}^{2} \bar{X}^{2}}{(s+\bar{X})^{3}}>0, \\
& \lambda_{1}=\beta \bar{X}-t_{1}-d_{2}-\frac{s p_{2} \bar{Y}}{s^{2}} \leq 0, \lambda_{2}=\frac{q p_{3} \bar{X}}{s+X}-\alpha \bar{Y}-t_{2}-d_{4} \leq 0,
\end{aligned}
$$

where $\bar{X}=d_{3} s /\left(q p_{1}-d_{3}\right)$ and $\bar{Y}=r s q\left(k q p_{1}-k d_{3}-d_{3} s\right) /$ $\left(q p_{1}-d_{3}\right)^{2} k$. Otherwise, the disease-free equilibrium point is asymptotically unstable.

Now let us see again the global stability analysis of model (8) around the endemic equilibrium point or positive equilibrium point $E^{*}\left(X^{*}, W^{*}, Y^{*}, Z^{*}, H^{*}\right)$ which shows coexistence. For that, let us state the following theorem and prove by taking appropriate Lyapunov function $L$.
Theorem 12 (global stability). Endemic equilibrium point $E^{*}$ $\left(X^{*}, W^{*}, Y^{*}, Z^{*}, H^{*}\right)$ exists and globally asymptotically stable.

Proof. Define appropriate Lyapunov function $L$ $(X, \quad W, \quad Y, \quad Z, \quad H)=(1 / 2)\left(X-X^{*}\right)^{2}+\left(\alpha_{1} / 2\right)$ $\left(W-W^{*}\right)^{2}+\left(\alpha_{2} / 2\right)\left(Y-Y^{*}\right)^{2}+\left(\alpha_{3} / 2\right)\left(Z-Z^{*}\right)^{2}+\left(\alpha_{4} / 2\right)$ $\left(H-H^{*}\right)^{2}$, where $\alpha_{1}, \alpha_{2}, \alpha_{3}, \alpha_{4}>0$ are chosen properly such that $d L / d t=0 \forall\left(X^{*}, W^{*}, Y^{*}, Z^{*}, H^{*}\right) \in \mathbb{R}_{+}^{5}$ and $d L / d t \leq 0 \forall($ $X, W, Y, Z, H) \in \mathbb{R}_{+}^{5}$. This implies $E^{*}$ of the system is Lyapunov stable and $d L / d t<0, \forall(X, W, Y, Z, H) \in \mathbb{R}_{+}^{5}$ near $E^{*}$. This implies $E^{*}$ is globally asymptotically stable point. Now differentiate the Lyapunov function $L$ with respect to $t$

$$
\begin{aligned}
\frac{d L}{d t}= & \left(X-X^{*}\right) \frac{d X}{d t}+\alpha_{1}\left(W-W^{*}\right) \frac{d I}{d t}+\alpha_{2}\left(Y-Y^{*}\right) \frac{d Y}{d t} \\
& +\alpha_{3}\left(Z-Z^{*}\right) \frac{d Z}{d t}+\alpha_{4}\left(H-H^{*}\right) \frac{d H}{d t} .
\end{aligned}
$$

Now substitute the model equation (8) into (58), we have the following equation:

$$
\begin{aligned}
\frac{d L}{d t}= & \left(X-X^{*}\right)\left[r X\left(1-\frac{X+W}{k}\right)+r_{1} H\right. \\
& \left.-\beta X W-\frac{p_{1} X Y}{s+X}-\frac{p_{3} X Z}{s+X}\right]+\alpha_{1}\left(W-W^{*}\right) \\
& \cdot\left[\beta X W-t_{1} W-d_{2} W-\frac{p_{2} W Y}{s+W}-\frac{p_{4} W Z}{s+W}\right] \\
& +\alpha_{2}\left(Y-Y^{*}\right)\left[q \frac{p_{1} X Y}{s+X}+q \frac{p_{2} W Y}{s+W}+r_{2} H-\alpha Y Z-d_{3} Y\right] \\
& +\alpha_{3}\left(Z-Z^{*}\right)\left[q \frac{p_{3} X Z}{s+X}+q \frac{p_{4} W Z}{s+W}+\alpha Y Z-t_{2} Z-d_{4} Z\right] \\
& +\alpha_{4}\left(H-H^{*}\right)\left[t_{1} W+t_{2} Z-d_{1} H-r_{1} H-r_{2} H\right] .
\end{aligned}
$$

Take out $X, W, Y, Z, H$ from each bracket and write a change as follows

$$
\begin{aligned}
\frac{d L}{d t}= & \left(X-X^{*}\right)\left(X-X^{*}\right)\left[r\left(1-\frac{X+W}{k}\right)+\frac{r_{1} H}{X}\right. \\
& \left.-\beta W-\frac{p_{1} Y}{s+X}-\frac{p_{3} Z}{s+X}\right]+\alpha_{1}\left(W-W^{*}\right)\left(W-W^{*}\right) \\
& \cdot\left[\beta X-t_{1}-d_{2}-\frac{p_{2} Y}{s+W}-\frac{p_{4} Z}{s+W}\right] \\
& +\alpha_{2}\left(Y-Y^{*}\right)\left(Y-Y^{*}\right) \\
& \cdot\left[q \frac{p_{1} X}{s+X}+q \frac{p_{2} W}{s+W}+\frac{r_{2} H}{Y}-\alpha Z-d_{3}\right] \\
& +\alpha_{3}\left(Z-Z^{*}\right)\left(Z-Z^{*}\right)\left[q \frac{p_{3} X}{s+X}+q \frac{p_{4} W}{s+W}+\alpha Y-t_{2}-d_{4}\right] \\
& +\alpha_{4}\left(H-H^{*}\right)\left(H-H^{*}\right)\left[\frac{t_{1} W}{H}+\frac{t_{2} Z}{H}-d_{1}-r_{1}-r_{2}\right] .
\end{aligned}
$$


By rearranging, it is obtained that

$$
\frac{d L}{d t}=-\left(X-X^{*}\right)^{2}[
$$

$\left.-r\left(1-\frac{X+W}{k}\right)-\frac{r_{1} H}{X}+\beta W+\frac{p_{1} Y}{s+X}+\frac{p_{3} Z}{s+X}\right]-\alpha_{1}\left(I-I^{*}\right)^{2}[-\beta X$ $\left.+t_{1}+d_{2}+\frac{p_{2} Y}{s+W}+\frac{p_{4} Z}{s+W}\right]-\alpha_{2}\left(Y-Y^{*}\right)^{2}\left[-q \frac{p_{1} s}{s+X}-q \frac{p_{2} W}{s+W}-\right.$ $\left.\frac{r_{2} H}{Y}+\alpha Z+d_{3}\right]-\alpha_{3}\left(Z-Z^{*}\right)^{2}\left[-q \frac{p_{3} X}{s+X}-q \frac{p_{4} W}{s+W}-\alpha Y+t_{2}+d_{4}\right.$

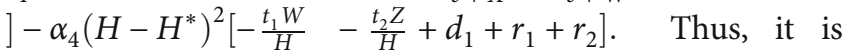
possible to set $\alpha_{1}, \alpha_{2}, \alpha_{3}, \alpha_{4}$ such that $d L / d t \leq 0$ and endemic equilibrium point $E^{*}$ is globally stable point.

\section{Basic Reproduction Number}

The basic reproduction number denoted by $R_{0}$ and defined as the expected number of people getting secondary infection among the whole susceptible population. This number shows a potential for spread of disease within a given population. When $R_{0}<1$, each infected individual produces on average less than one new infected individual so that the disease is expected to die out. On the other hand, if $R_{0}>1$, then each individual produces more than one new infected individual so that the disease is expected to continue spreading in the population.

Theorem 13. The basic reproduction number for infected prey at disease-free equilibrium point (DFEP) $\bar{E}(\bar{X}, 0, \bar{Y}, 0,0)=$ $\left(d_{3} s /\left(q p_{1}-d_{3}\right), \quad 0, \quad r s q\left(k q p_{1}-k d_{3}-d_{3} s\right) /\left(q p_{1}-d_{3}\right)^{2} k, 0\right.$, $0)$ is given by

$R_{01}=\frac{\left(q p_{1}-d_{3}\right)^{2} k \beta d_{3} s^{2}}{\left(q p_{1}-d_{3}\right)\left\{\left(q p_{1}-d_{3}\right)^{2} k s\left(t_{1}+d_{2}\right)+r s q p_{2}\left(k q p_{1}-k d_{3}-d_{3} s\right)\right\}}$.

Proof. Consider infected prey equation in (8), we have the following model equation

$$
\begin{aligned}
\frac{d W}{d t} & =\beta X W-t_{1} W-d_{2} W-\frac{p_{2} W Y}{s+W}-\frac{p_{2} W Z}{s+W} \\
& =\left(\beta X-\left[t_{1}+d_{2}+\frac{p_{2} Y}{s+W}+\frac{p_{2} Z}{s+W}\right]\right) W
\end{aligned}
$$

Now let us define functions $F$ and $V, F=\beta X, V=t_{1}+$ $d_{2}+p_{2} Y /(s+W)+p_{2} Z /(s+W)$.

Evaluate $F$ and $V$ at $\operatorname{DFEP} \bar{E}(\bar{X}, 0, \bar{Y}, 0,0)=$ $\left(d_{3} s /\left(q p_{1}-d_{3}\right), \quad 0, \quad r s q\left(k q p_{1}-k d_{3}-d_{3} s\right) /\left(q p_{1}-d_{3}\right)^{2} k, 0\right.$, $0)$,

$$
\begin{aligned}
F(\bar{E})= & \beta \bar{X}=\frac{\beta d_{3} s}{q p_{1}-d_{3}}, V(\bar{E})=t_{1}+d_{2}+\frac{p_{2} \bar{Y}}{s}=t_{1}+d_{2} \\
& +\frac{r s q p_{2}\left(k q p_{1}-k d_{3}-d_{3} s\right)}{\left(q p_{1}-d_{3}\right)^{2} k s} \\
= & \frac{\left(q p_{1}-d_{3}\right)^{2}\left(t_{1}+d_{2}\right) k s+r s q p_{2}\left(k q p_{1}-k d_{3}-d_{3} s\right)}{\left(q p_{1}-d_{3}\right)^{2} k s} .
\end{aligned}
$$

Then, the basic reproduction number of infected prey is

$$
\begin{aligned}
R_{01} & =F V^{-1} \\
& =\frac{\beta d_{3} s}{q p_{1}-d_{3}} * \frac{\left(q p_{1}-d_{3}\right)^{2} k s}{\left(q p_{1}-d_{3}\right)^{2}\left(t_{1}+d_{2}\right) k s+r s q p_{2}\left(k q p_{1}-k d_{3}-d_{3} s\right)}, \\
R_{01} & =\frac{\left(q p_{1}-d_{3}\right)^{2} k \beta d_{3} s^{2}}{\left(q p_{1}-d_{3}\right)\left\{\left(q p_{1}-d_{3}\right)^{2}\left(t_{1}+d_{2}\right) k s+r s q p_{2}\left(k q p_{1}-k d_{3}-d_{3} s\right)\right\}} .
\end{aligned}
$$

Theorem 14. The basic reproduction number for infected predators at disease-free equilibrium point (DFEP) $\bar{E}(\bar{X}, 0, \bar{Y}$,

$0,0)=\left(d_{3} s /\left(q p_{1}-d_{3}\right), \quad 0, \quad r s q\left(k q p_{1}-k d_{3}-d_{3} s\right) /\right.$ $\left.\left(q p_{1}-d_{3}\right)^{2} k, 0,0\right)$ is given by

$$
R_{02}=\frac{\left(q p_{1}-d_{3}\right)\left(q p_{3} d_{3}\right) k+\alpha r s q\left(k q p_{1}-k d_{3}-d_{3} s\right)}{\left(q p_{1}-d_{3}\right)^{2}\left(t_{2}+d_{4}\right) k}
$$

Proof. Consider the infected predator model equation in (8), we have the following equation

$$
\begin{aligned}
\frac{d Z}{d t} & =q \frac{p_{2} W Z}{s+W}+q \frac{p_{3} X Z}{s+W}+\alpha Y Z-t_{2} Z-d_{4} Z \\
& =\left[q \frac{p_{2} W}{s+W}+q \frac{p_{3} X}{s+W}+\alpha Y-\left(t_{2}+d_{4}\right)\right] Z .
\end{aligned}
$$

Now let us define functions $F$ and $V$ as $F=q\left(p_{2} W /(s\right.$ $+W))+q\left(p_{3} X /(s+W)\right)+\alpha Y, V=t_{2}+d_{4}$. Then, evaluate $F$ and $V$ at (DFEP) $\bar{E}(\bar{X}, 0, \bar{Y}, 0,0)=$ $\left(d_{3} s /\left(q p_{1}-d_{3}\right), \quad 0, \quad r s q\left(k q p_{1}-k d_{3}-d_{3} s\right) /\left(q p_{1}-d_{3}\right)^{2} k, 0\right.$, $0)$,

$$
\begin{aligned}
F(\bar{E}) & =\frac{q p_{3} d_{3} s}{s\left(q p_{1}-d_{3}\right)}+\frac{\alpha r s q\left(k q p_{1}-k d_{3}-d_{3} s\right)}{\left(q p_{1}-d_{3}\right)^{2} k} \\
& =\frac{\left(q p_{1}-d_{3}\right)\left(q p_{3} d_{3}\right) k+\alpha r s q\left(k q p_{1}-k d_{3}-d_{3} s\right)}{\left(q p_{1}-d_{3}\right)^{2} k}
\end{aligned}
$$

$$
V(\bar{E})=t_{2}+d_{4}
$$

Therefore, the basic reproduction number of infected predator is $R_{02}=F V^{-1}$, and hence,

$$
R_{02}=\frac{\left(q p_{1}-d_{3}\right)\left(q p_{3} d_{3}\right) k+\alpha r s q\left(k q p_{1}-k d_{3}-d_{3} s\right)}{\left(q p_{1}-d_{3}\right)^{2}\left(t_{2}+d_{4}\right) k} .
$$

\section{Simulation}

In this section, simulation of model (8) is carried out using DEDiscover version: 2.6.4. software. For simulation, a set of meaningful values are assigned to model parameters in Table 4, and initial values for model variables are given in Table 5. The model is arranged in such a way for simulation 
purposes.

$$
\begin{aligned}
\frac{d X}{d t}= & r * X *\left(1-\frac{X+W}{k}\right)+r^{-} 1 * H-\text { beta } * X * W \\
& -P^{-} 1 * X * \frac{Y}{s+X}-P^{-} 3 * X * \frac{Z}{s+X} / / \text { susceptible prey, } \\
\frac{d W}{d t}= & \text { beta } * X * W-t^{-} 1 * W-d^{-} 2 * W \\
& -P^{-} 2 * W * \frac{Y}{s+W}-P^{-} 4 * W * \frac{Z}{s+W} / / \text { infected prey, } \\
\frac{d Y}{d t}= & q * P^{-} 1 * X * \frac{Y}{s+X}+q * P^{-} 2 * W * \frac{Y}{s+W}+r^{-} 2 * H \\
& - \text { alpha } * Y * Z-d^{-} 3 * Y / / \text { susceptible predator, } \\
\frac{d Z}{d t}= & q * P^{-} 3 * X * \frac{Z}{s+X}+q * P^{-} 4 * W * \frac{Z}{s+W} \\
& - \text { alpha } * Y * Z-t^{-} 2 * Z-d^{-} 4 * Z / / \text { infected predator, } \\
\frac{d H}{d t}= & t^{-} 1 * W+t^{-} 2 * Z-d^{-} 1 * H-r^{-} 1 * H \\
& -r^{-} 2 * H / / \text { both infected populations under treatment. }
\end{aligned}
$$

From simulation in Figures 2 and 3, it can be concluded that treatment is a helpful tool to minimize or eradicate infection. It is shown that as the treatment rate increases on infected prey-predator, then the infected prey-predator population decreases rapidly. This shows due to the fact that the infected prey-predator population is recovering and moves to susceptible classes and that contributes to the susceptible prey-predator population to rise in number.

In the sample simulation in Figures 4 and 5, it is shown that high infection and predation results in the whole preypredator population declining to a certain level. Therefore, it is better to implement treatment mechanisms to sustain stability of the prey-predator system.

\section{Conclusions and Recommendation}

In this paper, It can be concluded that the formulated model is mathematically meaningful, valid, and biologically well posed by proving the boundedness, positivity, and existence of the solutions of the model. Trivial, axial, disease-free, and endemic equilibrium points are investigated. Moreover, It is observed that in our model, trivial equilibrium point is always locally asymptotically unstable. Axial equilibrium point is locally asymptotically stable if and only if the variables satisfy the following three conditions: (i) $\beta k-\left(t_{1}+d_{2}\right.$ ) $<0$, (ii) $q p_{1} k-d_{3}(s+k)<0$, and (iii) $q p_{3} k-\left(t_{2}+d_{4}\right)(s+k$ )$<0$.

Treatment is a helpful tool to minimize or eradicate infection in the prey-predator system. Therefore, providing treatment in an infected prey-predator system creates opportunity to recover from illness and the prey-predator population can be saved and exists in stable situation. Thus, it is recommended to apply treatment on infected prey-predator to make the whole prey-predator population safe and abundant in nature. One can extend this paper by assuming the predator grows logistically or by adding parameter like death rate on the prey or by including other variables like vaccination, immigration, migration on prey-predator system, and these things can be considered as limitation of this paper.

\section{Data Availability}

No primary data were used to support this study, and it is all secondary data.

\section{Conflicts of Interest}

The authors declare that there is no conflict of interest regarding the publication of this paper.

\section{References}

[1] S. Kumar and H. Kharbanda, "Stability analysis of preypredator model with infection, migration and vaccination in prey," 2017, http://arxiv.org/abs/1709.10319vl [math.DS].

[2] R. K. Naji and K. A. Hasan, "The dynamics of prey-predator model with disease in prey," Mathematics in Computer Science, vol. 2, no. 4, pp. 1052-1072, 2012.

[3] J. Chattopadhyay and O. Arino, "A predator-prey model with disease in the prey," Nonlinear Analysis, vol. 36, no. 6, pp. 747766, 1999.

[4] A. F. Bezabih, "Mathematical eco-epidemic model on preypredator system," IOSR Journal of Mathematics (IOSR-JM), vol. 16, no. 1, pp. 22-34, 2020.

[5] S. Sudipa, O. P. Misra, and J. Dhar, "Modeling a predator-prey system with infected prey in polluted environment," Applied Mathematical Modelling, vol. 34, no. 7, pp. 1861-1872, 2008.

[6] A. F. Bezabih, G. K. Edessa, and P. R. Koya, "Mathematical eco-epidemiological model on prey-predator system," Mathematical Modeling and Applications, vol. 5, no. 3, pp. 183190, 2020.

[7] A. F. Bezabih, G. K. Edessa, and P. R. Koya, "Mathematical epidemiology model analysis on the dynamics of COVID-19 pandemic," American Journal of Applied Mathematics, vol. 8, no. 5, pp. 247-256, 2020.

[8] S. P. Bera, A. Maiti, and G. Samanta, "A prey-predator model with infection in both prey and predator," Univerzitet $u$ Nišu, vol. 29, no. 8, pp. 1753-1767, 2015.

[9] Y. T. Hewindati, F. A. A. Faeldog, and F. A. Abdullah, "Dynamics of disease spread in a predator-prey system," Advanced Studies in Biology, vol. 6, no. 4, pp. 169-179, 2014.

[10] C. M. Silva, "Existence of periodic solutions for periodic ecoepidemic models with disease in the prey," Journal of Mathematical Analysis and Applications, vol. 453, no. 1, pp. 383397, 2017.

[11] A. Hugo, E. S. Massawe, and O. D. Makinde, "An ecoepidemiological mathematical model with treatment and disease infection in both prey and predator population," Journal of Ecology and Natural Environment, vol. 4, no. 10, pp. 266273, 2012.

[12] G. K. Edessa, B. Kumsa, and P. R. Koya, "Modeling and simulation study of the population dynamics of commensal-host- 
parasite system," American Journal of Applied Mathematics, vol. 6, no. 3, pp. 97-108, 2018.

[13] S. Tolcha, B. Kumsa, and P. R. Koya, "Modeling and simulation study of mutuality interactions with type II functional response and harvesting," American Journal of Applied Mathematics, vol. 6, no. 3, pp. 109-116, 2018.

[14] S. Saha and G. P. Samanta, "A prey-predator system with disease in prey and cooperative hunting strategy in predator," Journal of Physics A: Mathematical and Theoretical, vol. 53, no. 48 , article $485601,2020$.

[15] S. Sah, A. Maiti, and G. P. Samanta, "A predator-prey model with strong Allee effect and disease in prey population," International Journal of Ecological Economics and Statistics, vol. 40, no. 2, pp. 92-112, 2019.

[16] S. Sah, A. Maiti, and G. P. Samanta, "A Michaelis-Menten predator-prey model with strong Allee effect and disease in prey incorporating prey refuge," International Journal of Bifurcation and Chaos, vol. 28, no. 6, article 1850073, 2018.

[17] S. Saha and G. P. Samanta, "Analysis of a predator-prey model with herd behavior and disease in prey incorporating prey refuge," International Journal of Biomathematics, vol. 12, no. 1, article 1950007, 2019.

[18] M. Ashok, A. K. Pal, and G. P. Samanta, "On the dynamics of evolutionary Leslie-Gower predator-prey eco-epidemiological model with disease in predator," Ecological Genetics and Genomics, vol. 10, article 100034, 2019.

[19] S. Sharma and G. P. Samanta, "Analysis of a two prey one predator system with disease in the first prey population," International Journal of Dynamics and Control, vol. 3, no. 3, pp. 210-224, 2015.

[20] S. Sharma and G. P. Samanta, "A Leslie-Gower predator-prey model with disease in prey incorporating a prey refuge," Chaos, Solutions and Fractals, vol. 70, no. 1, pp. 69-84, 2015.

[21] M. Haque, "A predator-prey model with disease in the predator species only," Real World Applications, vol. 11, no. 4, pp. 2224-2236, 2010. 